\title{
Incoherent pair generation in a beam-beam interaction simulation
}

\author{
C. Rimbault* and P. Bambade \\ LAL, Orsay, France \\ K. Mönig \\ DESY, Zeuthen, Germany \\ D. Schulte \\ CERN, Geneva, Switzerland
}

(Received 25 November 2005; published 7 March 2006)

\begin{abstract}
This paper deals with two topics: the generation of incoherent pairs in two beam-beam simulation programs, GUINEA-PIG and CAIN, and the influence of the International Linear Collider (ILC) beam parameter choices on the background in the micro-vertex detector (VD) induced by direct hits. One of the processes involved in incoherent pair creation (IPC) is equivalent to a four fermions interaction and its cross section can be calculated exactly with a dedicated generator, BDK. A comparison of GUINEA-PIG and CAIN results with BDK allows to identify and quantify the uncertainties on IPC background predictions and to benchmark the GUINEA-PIG calculation. Based on this simulation and different VD designs, the five currently suggested ILC beam parameter sets have been compared regarding IPC background induced in the VD by direct IPC hits. We emphasize that the high luminosity set, as it is currently defined, would constrain both the choices of magnetic field and VD inner layer radius.
\end{abstract}

DOI: 10.1103/PhysRevSTAB.9.034402

PACS numbers: 41.60. $-\mathrm{m}, 41.75 .-\mathrm{i}, 29.27 . \mathrm{Fh}$

\section{INTRODUCTION}

GUINEA-PIG ${ }^{1}$ [1] and CAIN [2] are programs dedicated to simulating the beam-beam interaction in high-energy $e^{+} e^{-}$linear colliders such as International Linear Collider (ILC) and Compact Linear Collider (CLIC). Reliable and efficient computing tools are important to predict the luminosity and to study the backgrounds from secondary particles produced in the collisions, in order to optimize the design of both the machine and detectors. In the framework of the EuroTeV Design Study, ${ }^{2}$ a complete study, benchmarking, and improvement of GUINEA-PIG is under way, in collaboration with the team working on CAIN.

In this note, a study of the production of secondary $e^{+} e^{-}$ pairs created through the incoherent scattering of synchrotron radiation induced in the beam-beam interaction, socalled beamstrahlung, is presented. An important objective is to probe the realism of the predictions for the small fraction of such pairs which can reach the micro-vertex detector (VD). For large enough rates, the induced backgrounds can significantly confuse the pattern recognition for associating hits in the VD, and hence lead to reduced impact parameter resolution for the tracks involved. The aims of the study are to identify and assess the following:

(i) The intrinsic theory uncertainty in the prediction; for this, the three physical processes which contribute to $e^{+} e^{-}$

\footnotetext{
*Electronic address: rimbault@lal.in2p3.fr

${ }^{1}$ Generator of unwanted interactions for numerical experiment analysis program, interfaced to GEANT.

${ }^{2}$ European design study towards a global $\mathrm{TeV}$ linear collider.
}

pair production are investigated and compared for the different settings available in GUINEA-PIG and CAIN. An additional dedicated four-fermion event generator, BDK [3], based on a different theoretical approach, has also been used for one of the physical processes,

(ii) The changes in rates reaching the VD for the different ILC beam parameter sets [4] proposed as alternatives to the nominal reference, and for the most relevant design parameters of the three detector concepts presently under study [5-7].

\section{INCOHERENT PAIR CREATION PROCESSES}

Following the emission of beamstrahlung photons, $e^{+} e^{-}$ pairs are produced both by coherent (CPC) and incoherent pair creation (IPC) processes. The CPC process consists of the interaction of the beamstrahlung photons with the collective electromagnetic field of the opposite beam, while the IPC pairs arise from the interaction of both real or virtual photons from each beam with individual particles of the other beam. For ILC running energies up to $1 \mathrm{TeV}$ in the center of mass, the coherent production process is negligible compared to the incoherent one.

Three main physical processes are responsible for IPC, referred to, respectively, as Breit-Wheeler (BW), BetheHeitler (BH), and Landau-Lifshitz (LL). Two real photons interact in the $\mathrm{BW}$ process, a real and a virtual in the $\mathrm{BH}$ process and two virtual ones in the LL process. The real photons are from the beamstrahlung and virtual ones can be pictured as an off-shell photon cloud accompanying each high-energy beam electron or positron. While both GUINEA-PIG and CAIN use the explicit leading-order cross- 


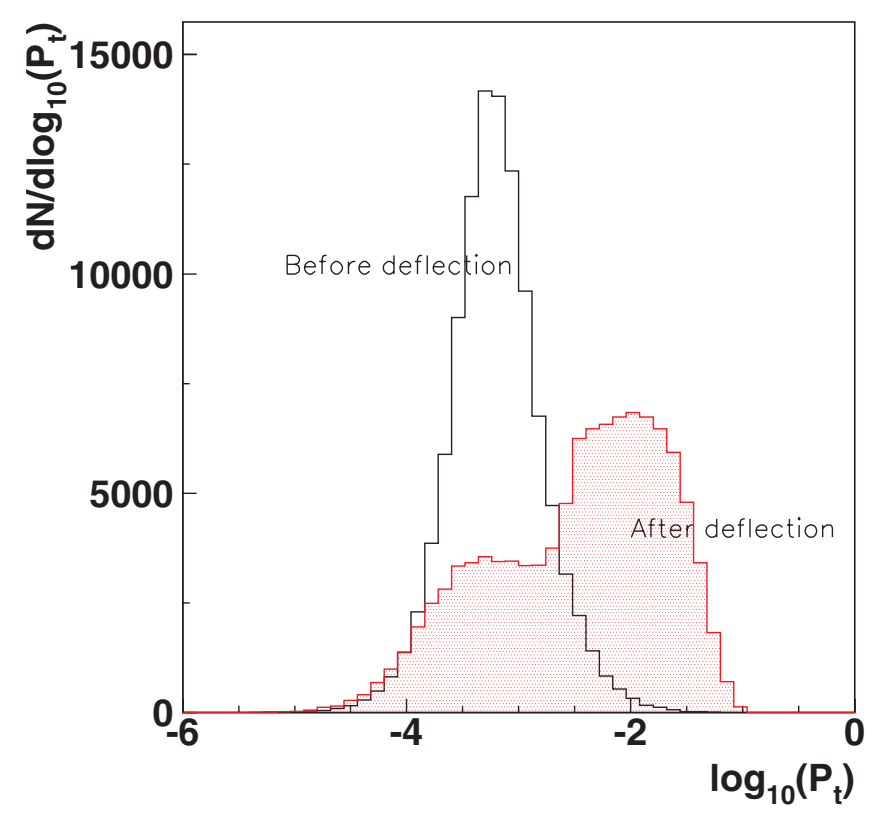

FIG. 1. (Color) $\log _{10}\left(P_{t}\right)$ distribution of the pairs per bunch crossing before (empty histogram) and after (solid histogram) electromagnetic deflections from the opposite beam, $P_{t}$ being in $\mathrm{GeV} / c$.

section calculation for the BW process, an approximation is used for the processes involving virtual photons, called the equivalent photon approximation. This approximation treats virtual photons as real ones by convoluting an equivalent spectrum for the virtual photons with the cross section for the real-real case. These photons are treated as being real as long as their virtuality remains below an upper limit, above which they are ignored. This upper limit, $Q_{\max }^{2}$, is fixed to the electron mass squared, $m_{e}^{2}$, in CAIN whereas GUINEA-PIG offers the choice between $m_{e}^{2}$, $m_{e}^{2}+p_{\perp}^{2}$, the transverse mass squared of the final state and $s / 4$, half of the invariant mass squared. The latest choice is recommended and used by default in GUINEA-PIG. More details can be found in $[1,8]$.

Two important phenomena must be taken into account in the production of secondary pairs from the beam-beam interaction: deflections due to the electromagnetic field of the opposite beam and the so-called "beam-size effect."

(i) Electromagnetic deflections: Most pairs are produced with very small angles along the beam axis. Half of them are emitted in the direction opposite to the beam of same charge and are consequently defocused while those following the direction of the beam of same charge are focused. The resulting effect is shown both in Figs. 1 and 2. A clear accumulation at large transverse momenta can be seen. Electromagnetic deflections however do not affect the energy spectrum or the overall production cross section. An additional effect which arises from electromagnetic deflections is a suppression of virtual-photon emissions at small angles.

(ii) Suppression from finite beam size: The virtuality of the quasireal photons involved in two of the IPC processes implies some spatial indeterminacy. For the smallest virtual-photon transverse momenta, this quantum uncertainty on their localization can exceed the physical sizes of the tightly focused colliding beams. A suppression of the cross section for small transverse momentum virtual photons is expected from this and consequently a reduction in pair production rates [9].

\section{A. Comparison of GUINEA-PIG and CAIN 1. Input parameters and options}

Table I gives the values of the beam parameters for several ILC design optimizations under consideration [4]. The American version of the cold accelerator design (USSC) has been used to compare GUINEA-PIG and CAIN. The parameters of the TeV Energy Superconducting Linear
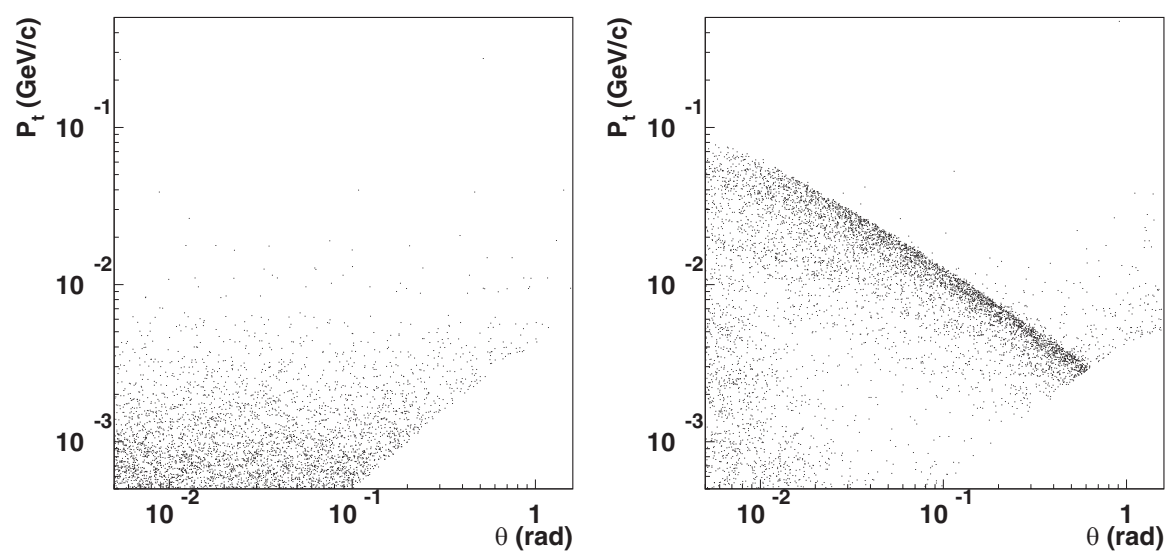

FIG. 2. $\quad P_{t}$ versus $\theta$ distributions for IPC particles before (left-hand plot) and after (right-hand plot) electromagnetic deflections from the opposite beam. 
TABLE I. Beam parameters for different ILC design optimizations under consideration. $E_{c m}$ is the center of mass energy of the collision, $N$ the number of particles per bunch, $N_{b}$ the number of bunches per train, $\beta$ are the beta functions at the interaction point, $\gamma \epsilon$ are the normalized emittances, and $\sigma$ are the bunch dimensions at the interaction point.

\begin{tabular}{lccccccc}
\hline \hline & TESLA & USSC & Nominal & Low $Q$ & Large $Y$ & Low $P$ & High Lum \\
\hline$E_{\mathrm{cm}}[\mathrm{GeV}]$ & 500 & 500 & 500 & 500 & 500 & 500 & 500 \\
$N\left[10^{10}\right]$ & 2 & 2 & 2 & 1 & 2 & 2 & 2 \\
$N_{b}$ & 2820 & 2820 & 2820 & 5640 & 2820 & 1330 & 2820 \\
$\beta_{x}[\mathrm{~mm}]$ & 15.0 & 15.0 & 21.0 & 12.0 & 10.0 & 10.0 & 10.0 \\
$\beta_{y}[\mathrm{~mm}]$ & 0.4 & 0.4 & 0.4 & 0.2 & 0.4 & 0.2 & 0.2 \\
$\gamma \epsilon_{x}\left[10^{-6} \mathrm{mrad}\right]$ & 10.0 & 9.6 & 10.0 & 10.0 & 12.0 & 10.0 & 10.0 \\
$\gamma \epsilon_{y}\left[10^{-6} \mathrm{mrad}\right]$ & 0.030 & 0.040 & 0.040 & 0.030 & 0.080 & 0.035 & 0.030 \\
$\sigma_{x}[\mathrm{~nm}]$ & 554 & 543 & 655 & 495 & 495 & 452 & 452 \\
$\sigma_{y}[\mathrm{~nm}]$ & 5.0 & 5.7 & 5.7 & 3.5 & 8.1 & 3.8 & 3.5 \\
$\sigma_{z}[\mu \mathrm{m}]$ & 300 & 300 & 300 & 150 & 500 & 200 & 150 \\
\hline \hline
\end{tabular}
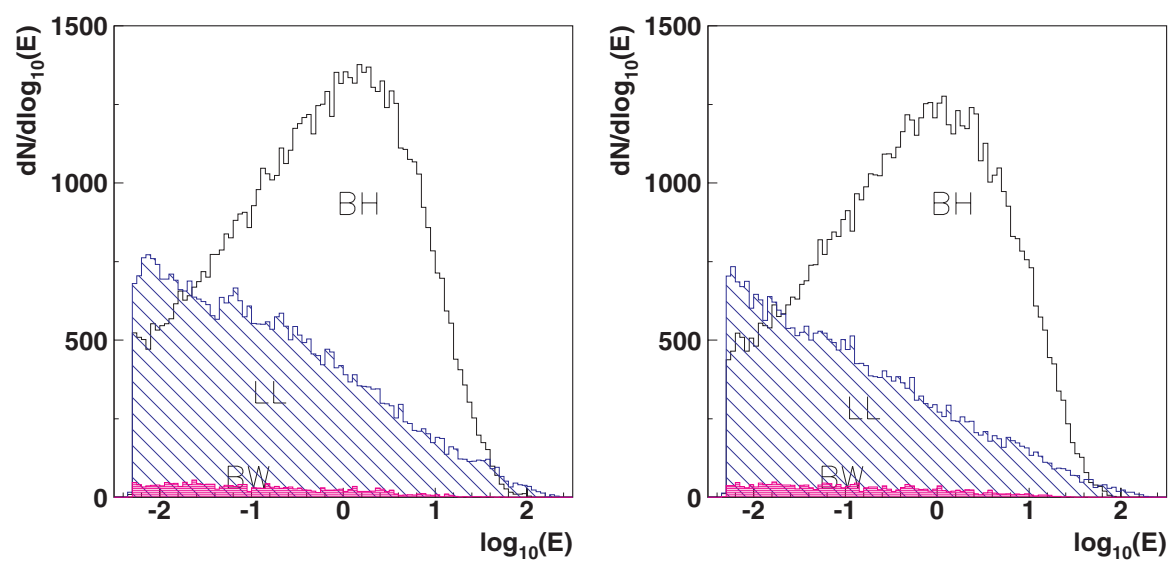

FIG. 3. (Color) $\log _{10}(E)$ spectra for the three incoherent pair creation processes, BH, LL, and BW modeled in GUINEA-PIG (left plot) and CAIN (right plot), $E$ being in GeV.

Accelerator (TESLA) Technical Design Report (TDR) are also shown for Ref. [5]. A cut of $5 \mathrm{MeV}$ is applied on the energy of the produced IPC electron or positron. The default settings of GUINEA-PIG and CAIN are first used

(i) The suppression effect from the finite beam size is activated in both programs.

(ii) The virtual-photon suppression effect due to the field of the opposite beam is turned off.

(iii) The maximum virtuality scale allowed in pair production processes is set to the electron mass and to half the center of mass energy of the scattering, respectively, in CAIN and GUINEA-PIG. All the results are given for one bunch crossing.

Qualitatively speaking, CAIN and GUINEA-PIG give very similar results. Energy spectra are shown in Fig. 3 for BW, $\mathrm{BH}$, and LL processes, and show a very good agreement between the two simulations. One notes that the mixed process is largely dominant whereas the real-real process represents only a few percent of the total production. Similar conclusions hold for the $\left(P_{t}, \theta\right)$ spectra obtained with both programs (see Fig. 4 and the plot on the left-hand side of Fig. 2).

The cross sections ${ }^{3}$ and the corresponding numbers of secondary electrons produced per bunch crossing for each IPC process are given in Table II in the case of the USSC parameters. CAIN predicts about $12 \%$ fewer particles than GUINEA-PIG. This comes entirely from the processes which involve virtual photons, especially the virtual-virtual one for which one finds a $20 \%$ difference.

\section{B. Comparison with BDK for the Landau-Lifshitz process}

BDK is a Monte-Carlo event generator for four-fermion processes in $e^{+} e^{-}$interactions which is based on complete calculations with leading-order massive matrix elements for all relevant electroweak diagrams involved. The results

\footnotetext{
${ }^{3}$ For the two processes involving real photons, $\mathrm{BW}$ and $\mathrm{BH}$, the quoted cross sections should be understood as effective ones.
} 


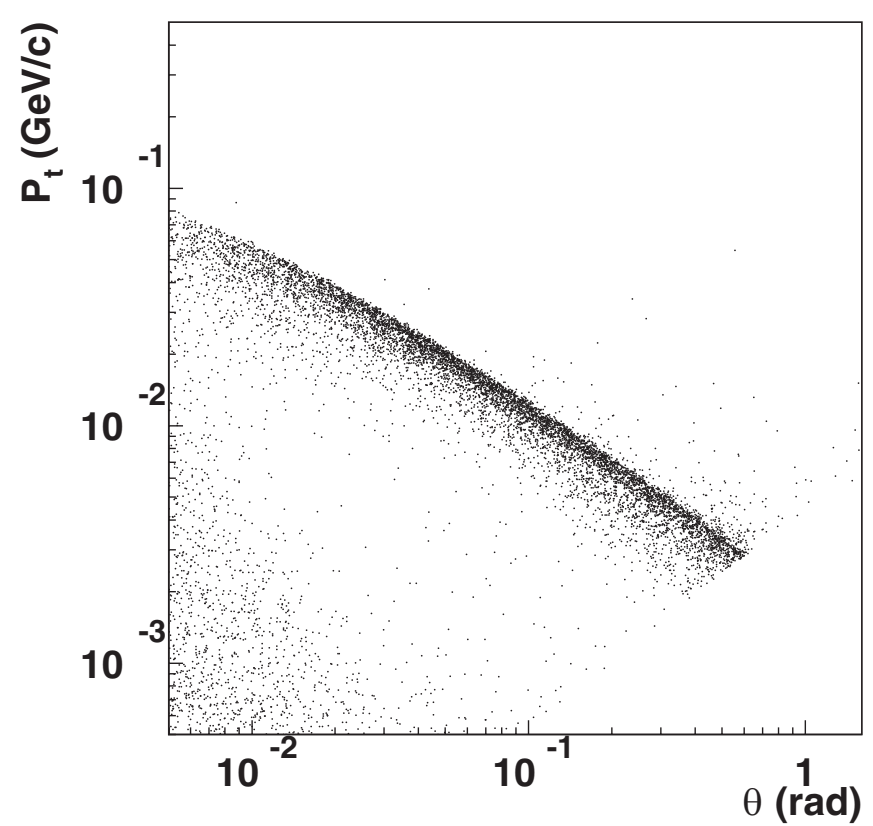

FIG. 4. Transverse momentum, $P_{t}$, versus polar angle, $\theta$, for $e^{ \pm}$from IPC processes modeled in CAIN.

for the $e^{+} e^{-} \rightarrow e^{+} e^{-} e^{+} e^{-}$process obtained in GUINEAPIG and CAIN can be compared with BDK as a test of the equivalent photon approximation used in the two beambeam simulations.

To perform this test, one has to turn off the cross-section suppression from the finite beam size, used by default in GUINEA-PIG and CAIN and look at the distributions of pair space variables before their deflection. In BDK, in order to generate the same phase space as in GUINEA-PIG and CAIN, the square of the invariant mass of the produced pairs is lowered down to $10^{-6} \mathrm{GeV}^{2}$, corresponding to the threshold for electron-pair production.

The log-energy, transverse momentum and polar angle distributions of the IPC particles produced through the Landau-Lifshitz process are shown in Figs. 5 and 6, re-

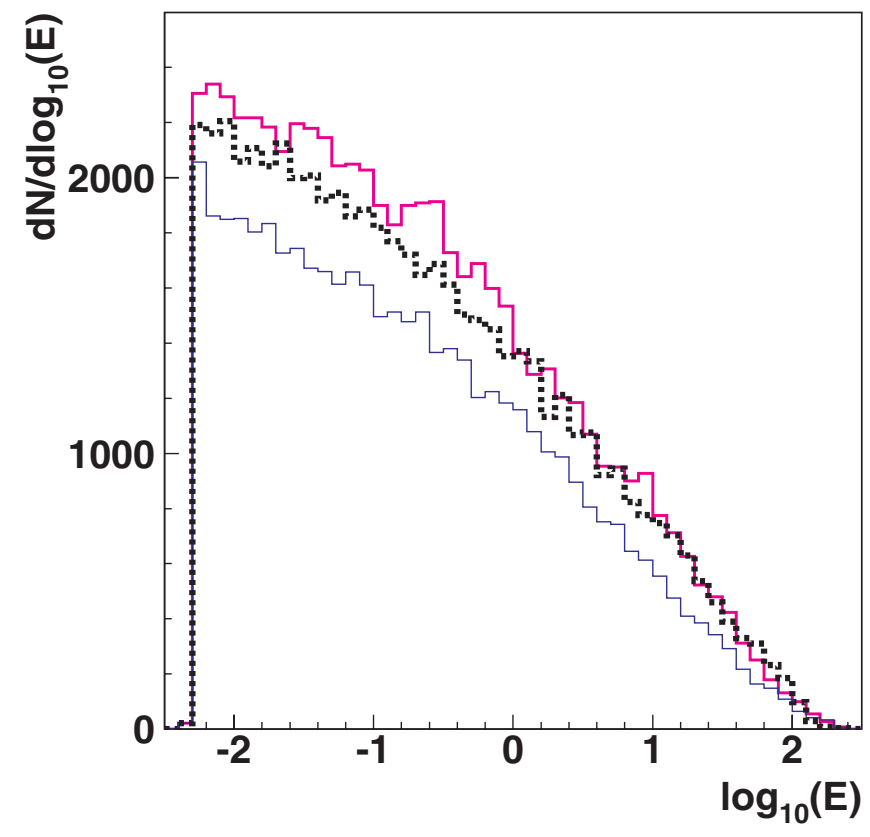

FIG. 5. (Color) $\log _{10}(E)$ spectrum for the Landau-Lifshitz process in GUINEA-PIG (upper line), CAIN (lower thin line) and BDK (dashed line), $E$ being in $\mathrm{GeV}$.

spectively. Table III gives the cross sections for the pairs produced through all processes, without the suppression from the finite beam size. Comparing with the results in Table II, for which this effect was included, one can see that the reduction induced amounts to about $40 \%$ and that it of course only is present for processes involving virtual photons. Both GUINEA-PIG and CAIN predict LandauLifshitz cross sections in broad agreement with BDK, with GUINEA-PIG being the closest.

\section{Event rates in the micro-vertex detector}

A small fraction of the electrons and positrons produced through IPC processes can reach the micro-vertex detector

TABLE II. Number of particles per bunch crossing and (effective) cross sections for each IPC process in GUINEA-PIG and CAIN.

\begin{tabular}{lcc}
\hline \hline & GUINEA-PIG & CAIN \\
\hline Cll processes & 58.0 & \\
Breit-Wheeler & 1.05 & 50.7 \\
Bethe-Heitler & 37.7 & 1.04 \\
Landau-Lifshitz & 19.2 & 34.5 \\
\hline \multicolumn{1}{c}{ Total number of electrons and positrons per bunch crossingwith $L=1.82 \mu \mathrm{b}^{-1}$} \\
\hline All processes & 105500 & 92300 \\
Breit-Wheeler & 1900 & 1900 \\
Bethe-Heitler & 68600 & 62800 \\
Landau-Lifshitz & 35000 & 27700 \\
\hline \hline
\end{tabular}



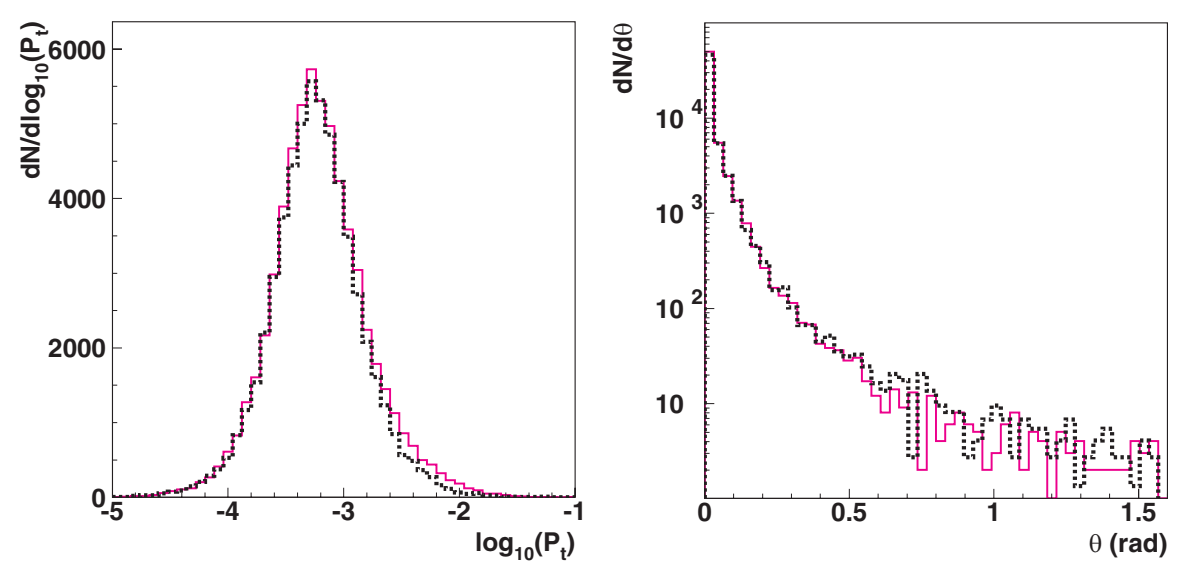

FIG. 6. (Color) $\log _{10}\left(P_{t}\right)$ with $P_{t}$ in $\mathrm{GeV} / c$ (left plot) and polar angle (right plot) distributions for the Landau-Lifshitz process in GUINEA-PIG and BDK (dashed line).

(VD). The corresponding rates are computed for a detector consisting of five cylindrical layers [5], $L_{\mathrm{i}}, i=1-5$, with the following lengths, $\ell$ and radii, $r$ :

$$
\begin{gathered}
\quad \ell_{1}=100 \mathrm{~mm}, \quad \ell_{2-5}=250 \mathrm{~mm}, \\
r_{1}=15 \mathrm{~mm}, \quad r_{2}=26 \mathrm{~mm}, \quad r_{3}=37 \mathrm{~mm}, \\
r_{4}=48 \mathrm{~mm}, \quad r_{5}=60 \mathrm{~mm} .
\end{gathered}
$$

To determine if a particle with transverse momentum $P_{t}$ and production angle $\theta_{0}$ traveling in a magnetic field $B$ reaches the $\mathrm{VD}$, the helix equation is used:

$$
r(z)=r_{0} \sqrt{2(1-\cos \phi(z))}, \quad \phi(z)=\frac{z}{r_{0}} \tan \theta_{0},
$$

with

$$
r_{0}[m]=3.33 P_{t}[\mathrm{GeV} / \mathrm{c}] / B[T],
$$

where $r(z)$ is the distance in meters to the beam axis at the abscissa $z$. The magnetic field is set to $4 \mathrm{~T}$ and the USSC parameters are still used.

Figure 7 highlights the region in the two-dimensional $\left(P_{t}, \theta\right)$ distribution corresponding to pairs which can reach the VD. The minimum transverse momentum and polar angle involved is found to be $P_{t}>5 \mathrm{MeV}$ and $\theta>10^{\circ}$.

This region is fortunately outside of the beam-beam deflection induced accumulation zone, for the beam pa-

TABLE III. Cross sections for incoherent pair production without finite beam-size suppression effects in GUINEA-PIG, CAIN, and BDK

\begin{tabular}{lccc}
\hline \hline$\sigma(\mathrm{mb})$ & GUINEA-PIG & CAIN & BDK \\
\hline All processes & 101 & 89.5 & \\
Breit-Wheeler & 1.01 & 1.11 & \\
Bethe-Heitler & 66.3 & 61.7 & \\
Landau-Lifshitz & 33.9 & 26.7 & 31.8 \\
\hline \hline
\end{tabular}

rameters considered. The corresponding $P_{t}$ and $\theta$ are not a consequence of the electromagnetic deflections, but arise intrinsically in the IPC process, as shown in Fig. 8.

It is important to note that the ranges in $P_{t}$ and $\theta$ shown in Fig. 7, corresponding to pairs which can reach the VD, depend both on the VD geometrical design (for $\theta$ ) and on the detector magnetic field (for $P_{t}$ ). On the other hand, the exact location of the beam-beam deflection induced accumulation zone depends on the chosen ILC beam parameters. These considerations will be developed further in Sec. III.

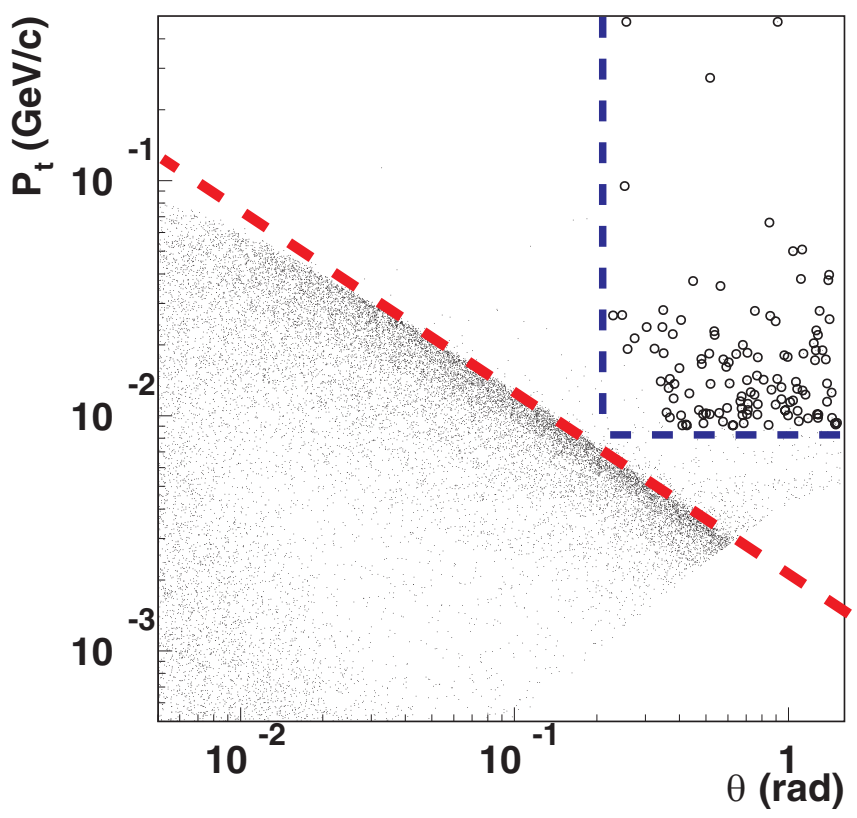

FIG. 7. (Color) Distribution of $P_{t}$ versus $\theta$ for electrons from IPC processes. The region corresponding to particles reaching the VD (circles) is indicated with the two dashed lines for the detector configuration described in the text. A thick dotted line highlights the edge of the beam-beam deflection induced accumulation zone. 


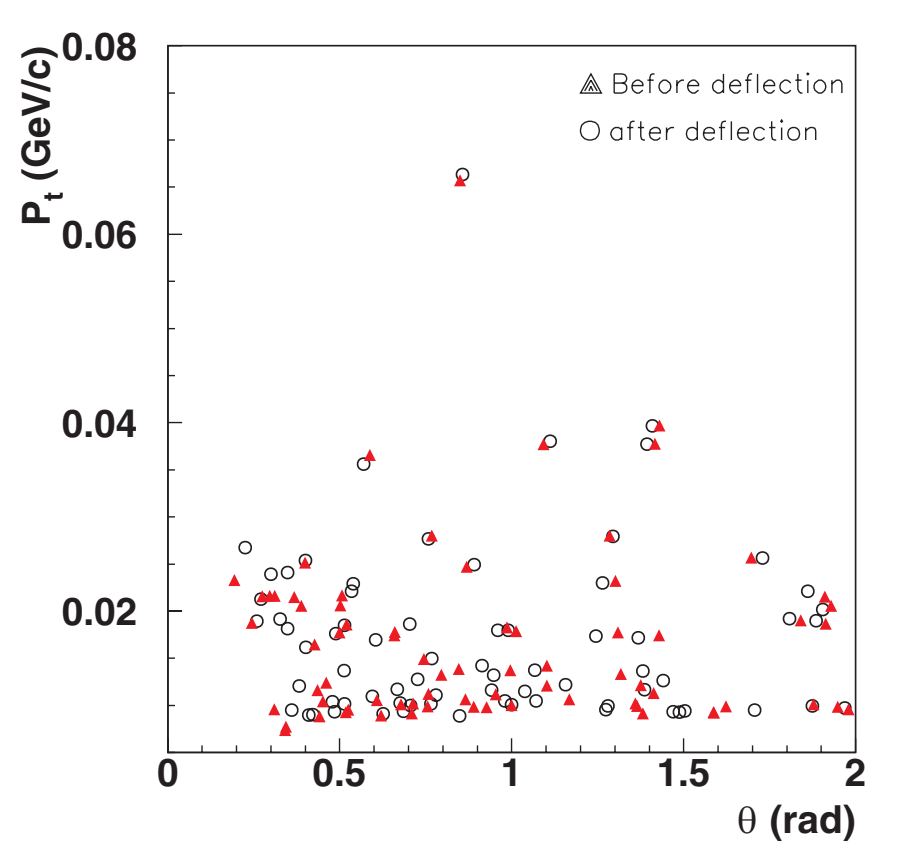

FIG. 8. (Color) Distribution of $P_{t}$ versus $\theta$ for electrons from IPC processes reaching the VD, before electromagnetic deflections (triangles) and after (circles).

Table IV gives the cross section of the pair background reaching the VD predicted by GUINEA-PIG and CAIN, with (upper lines) and without (lower lines) the expected suppression from the finite beam size. It can be seen that the beam-size effect has little influence on the background rates in the VD (within statistical fluctuations). Activating the virtual-photon suppression effect due to the opposite beam field has also been tried (instead of the beam-size effect, to avoid potential double counting) and leads to a VD background reduction of the order of $10 \%$.

There is at least a $40 \%$ difference between CAIN and GUINEA-PIG for the total number of IPC particles reaching the VD. A comparison with BDK for the Landau-Lifshitz process indicates that CAIN seems to underestimate the corresponding rate by a factor 3 , whereas GUINEA-PIG is in good agreement.

TABLE IV. Cross sections for the pair background reaching the VD predicted by GUINEA-PIG, CAIN, and BDK, with (upper lines) and without (lower lines) the "beam-size effect."

\begin{tabular}{lrrr}
\hline \hline$\sigma(\mu \mathrm{b})$ & GUINEA-PIG & \multicolumn{1}{c}{ CAIN } & BDK \\
\hline All processes & $64.1 \pm 5.9$ & $37.4 \pm 4.5$ & \\
& $60.5 \pm 6.0$ & $36.5 \pm 4.5$ & \\
Breit-Wheeler & $8.2 \pm 2.1$ & $6.4 \pm 1.9$ & \\
& $10.3 \pm 2.4$ & $7.0 \pm 2.0$ & \\
Bethe-Heitler & $26.6 \pm 3.8$ & $20.9 \pm 3.3$ & \\
& $20.5 \pm 3.3$ & $16.6 \pm 3.0$ & \\
Landau-Lifshitz & $29.3 \pm 4.0$ & $10.2 \pm 2.3$ & \\
& $29.7 \pm 4.0$ & $13.4 \pm 2.7$ & $37.5 \pm 5.3$ \\
\hline \hline
\end{tabular}

TABLE V. Cross sections for incoherent pair production, $\sigma_{e^{+} e^{-}}$, and for the pair background reaching the VD, $\sigma_{V D}$, predicted by GUINEA-PIG, with the "beam-size effect" activated and using $Q_{\max }=m_{e}$ for the maximum virtuality in the equivalent photon spectrum instead of the default value.

\begin{tabular}{lcr}
\hline \hline GUINEA-PIG & $\sigma_{e^{+} e^{-}}(\mathrm{mb})$ & $\sigma_{V D}(\mu \mathrm{b})$ \\
\hline All processes & 51.8 & $32.0 \pm 4.3$ \\
Breit-Wheeler & 1.09 & $5.7 \pm 1.8$ \\
Bethe-Heitler & 35.2 & $16.5 \pm 3.1$ \\
Landau-Lifshitz & 15.6 & $9.7 \pm 2.4$ \\
\hline \hline
\end{tabular}

\section{Origin of the difference between CAIN and GUINEA-PIG}

This difference between CAIN and GUINEA-PIG can be traced to the different choices made in the two programs for the maximum virtuality, $Q_{\max }$, used in the equivalent photon spectrum (see Sec. II). Table V shows this: if $Q_{\max }$ is set to $m_{e}$ in GUINEA-PIG instead of the default value, one obtains $\sigma_{\text {all }}=32.0 \mu \mathrm{b}$ and $\sigma_{L L}=9.7 \mu \mathrm{b}$ for the background reaching the VD, which is indeed consistent with the results from CAIN (see Tables II and IV).

In order to assess the importance of cutting events with photon virtualities beyond a certain value as part of the equivalent photon approximation, the virtuality spectrum generated in GUINEA-PIG, using the default running option $Q_{\max }^{2}=s / 4$ and no beam-size effect, was extracted and compared with that which can be reconstructed from BDK events using the following equation:

$$
\begin{aligned}
Q^{2} & =\left(P_{i}-P_{s}\right)^{2} \\
& \simeq m_{e}^{2}\left(2-\left(E_{i} / E_{s}+E_{s} / E_{i}\right) \cos \theta\right)-2 E_{i} E_{s}(1-\cos \theta),
\end{aligned}
$$

with $\theta \ll 1$,

where $P_{i, s}=\left(E_{i, s}, \overrightarrow{p_{i, s}}\right)$ are the four-momenta of the incident and scattered electrons and $\theta$ the scattering angle. The comparison is shown in Fig. 9, where the position of the electron mass is also indicated.

A much better agreement between the spectra in GUINEA-PIG and BDK can be seen with the GUINEA-PIG default choice for the maximum photon virtuality cut, at half the invariant mass of the process, than when setting it to $m_{e}$ as in CAIN.

Figure 10 shows the virtualities of the two photons involved in the LL process, reconstructed from the BDK events. The highlighted squares correspond to the events for which a pair particle reaches the VD. The dashed lines correspond to the electron mass. $24 \%$ of the produced pairs arise through an interaction where at least one of the quasireal photons involved has a virtuality larger than $m_{e}$. Removing these events reduces the cross section by about $24 \mathrm{mb}$, which corresponds to the CAIN result (see Table III). Moreover, only 33\% of the VD background comes from two low virtuality photons, which corresponds 


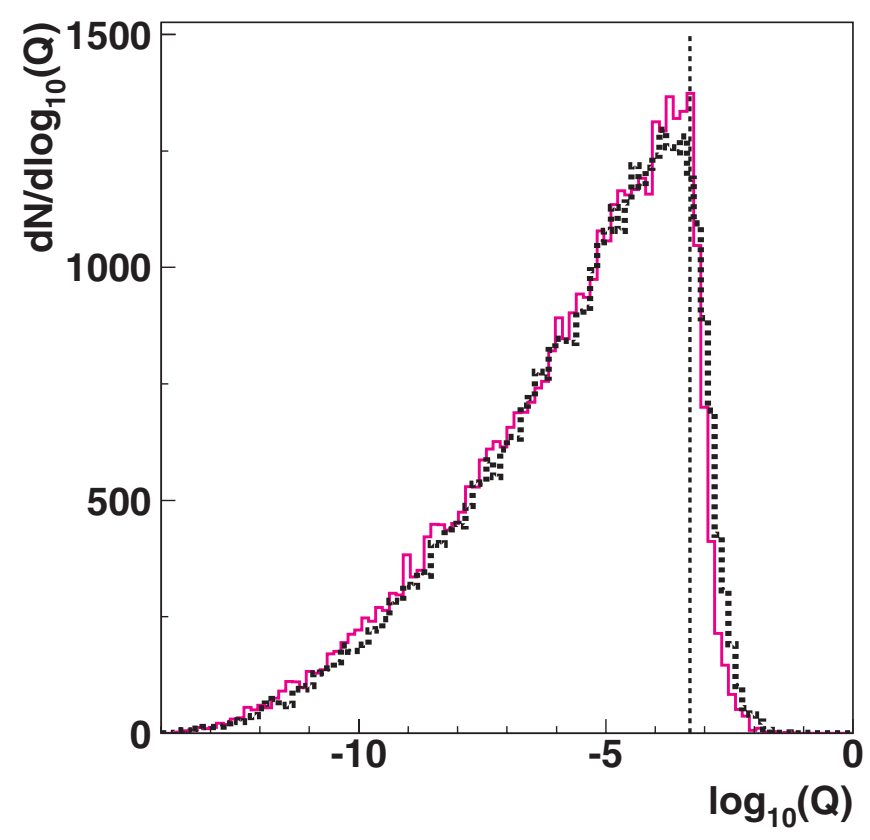

FIG. 9. (Color) Comparison of GUINEA-PIG and BDK (dashed thick line) photon virtuality decimal logarithm spectra, $Q$ being in $\mathrm{GeV}$. The vertical dashed line indicates the position of the electron mass.

to a cross section of $12 \mu \mathrm{b}$, again in accordance with CAIN (see Table IV).

BDK being a genuine matrix element calculation, it should give the more reliable prediction at large virtuality.

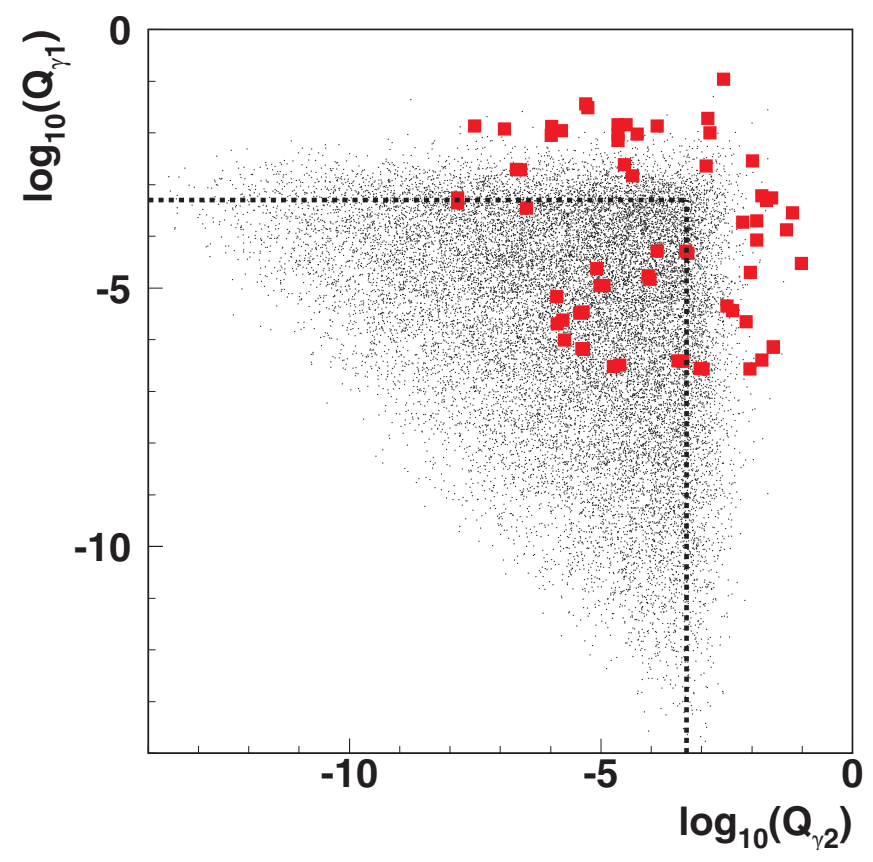

FIG. 10. (Color) $\log _{10}\left(Q_{\gamma_{1}}\right)$ versus $\log _{10}\left(Q_{\gamma_{2}}\right)$ in BDK simulation, $Q_{\gamma i}$ being in $\mathrm{GeV}$. The highlighted points correspond to events with pair particles reaching the VD. The dashed lines indicate the position of the electron mass.
On the other hand, the equivalent photon approximation should be best in the quasireal limit. Since the two spectra have very similar shapes and since the additional cross section predicted by BDK, as compared with the equivalent photon approximation using a cut on photon virtualities at $m_{e}$, is in the large virtuality part of the spectrum, it can be argued that the GUINEA-PIG prediction, with the cut set at its default value of half the invariant mass of the produced final state, is the better one. How much this prediction can be trusted is however limited by the fact that this default cut, although it seems a rather natural choice, is ad hoc, while the matrix elements used in BDK are only leadingorder ones. Nonetheless, what does seem clear is that the cross section predicted by the equivalent photon approximation with a cut on photon virtualities at $m_{e}$ is too small.

\section{IMPACT OF ILC BEAM PARAMETER SETS ON PAIR BACKGROUND RATES IN THE VERTEX DETECTOR}

Looking at Fig. 7, two regions are interesting to highlight: that corresponding to events which can reach the VD, represented by the rectangle, and the stripe where most events accumulate after being deflected by the electromagnetic field of the opposite beam. The edge of this stripe is represented by the thick dashed line.

The acceptance rectangle depends on the characteristics of the VD (length and radius) and on the magnetic field, B, while the accumulation stripe depends on the beam parameters $\left(\sigma_{x}, \sigma_{z}, N\right)$. One has to be sure that the chosen beam parameters and VD design allows the VD acceptance rectangle to stay clear off the pair accumulation stripe. Situations in which this stripe overlaps the VD acceptance are referred to in the following as "inflationary case."

We have tested the five official sets of beam parameters: nominal, low $Q$, large $Y$, low $P$, and high luminosity, for three values of the magnetic field, 3T [7], 4T [5], and 5T [6], and four different inner VD layer radii, 10, 15, 20, and $25 \mathrm{~mm}$. Results using the TESLA TDR configuration are also given for reference and comparison, since it corresponds to a case which was studied in detail with full GEANT-based detector simulations [5]. GUINEA-PIG was used with its default settings as previously described.

Table VI gives the cross sections for the IPC particles reaching the VD for all the studied cases. The underlined numbers correspond to inflationary cases. For all such cases, the smallest VD inner layer radii are probably excluded.

Increasing the magnetic field by $1 \mathrm{~T}$ for a given $\mathrm{VD}$ design $(15$ or $20 \mathrm{~mm})$ suppresses on average $38 \%$ of the considered background. One can also notice an approximate equivalence between the following cases:

(i) $\left(r_{1}=20 \mathrm{~mm}, B=4 \mathrm{~T}\right)$ and $\left(r_{1}=15 \mathrm{~mm}, B=5 \mathrm{~T}\right)$,

(ii) $\left(r_{1}=20 \mathrm{~mm}, B=3 \mathrm{~T}\right)$ and $\left(r_{1}=15 \mathrm{~mm}, B=\right.$ $4 \mathrm{~T})$, 
TABLE VI. Cross sections in $\mu \mathrm{b}$ for the pair background reaching the VD per bunch crossing for different beam parameters sets. The TESLA TDR case is shown for reference.

\begin{tabular}{|c|c|c|c|c|c|c|}
\hline$\sigma[\mu \mathrm{b}]$ & $3 \mathrm{~T}$ & $4 \mathrm{~T}$ & $5 \mathrm{~T}$ & $3 \mathrm{~T}$ & $4 \mathrm{~T}$ & $5 \mathrm{~T}$ \\
\hline & \multicolumn{3}{|c|}{ TESLA TDR } & \multicolumn{3}{|c|}{ Nominal } \\
\hline $\begin{array}{l}r_{1}=10 \mathrm{~mm} \\
r_{1}=15 \mathrm{~mm} \\
r_{1}=20 \mathrm{~mm} \\
r_{1}=25 \mathrm{~mm}\end{array}$ & $\begin{array}{l}\frac{1320}{85 \pm 7} \\
46 \pm 5 \\
34 \pm 4\end{array}$ & $\begin{array}{l}171 \pm 9 \\
49 \pm 5 \\
27 \pm 4 \\
19 \pm 3\end{array}$ & $\begin{array}{l}90 \pm 7 \\
30 \pm 4 \\
17 \pm 3 \\
11 \pm 2\end{array}$ & $\begin{array}{l}\underline{1067} \\
88 \pm 8 \\
58 \pm 6 \\
42 \pm 5\end{array}$ & $\begin{array}{c}166 \pm 11 \\
59 \pm 6 \\
36 \pm 5 \\
23 \pm 4\end{array}$ & $\begin{array}{l}92 \pm 8 \\
39 \pm 5 \\
21 \pm 4 \\
16 \pm 3\end{array}$ \\
\hline & \multicolumn{3}{|c|}{ Low $Q$} & \multicolumn{3}{|c|}{ Large $Y$} \\
\hline $\begin{array}{l}r_{1}=10 \mathrm{~mm} \\
r_{1}=15 \mathrm{~mm} \\
r_{1}=20 \mathrm{~mm} \\
r_{1}=25 \mathrm{~mm}\end{array}$ & $\begin{array}{c}\underline{600} \\
90 \pm 11 \\
53 \pm 9 \\
38 \pm 7\end{array}$ & $\begin{array}{c}143 \pm 14 \\
55 \pm 9 \\
31 \pm 7 \\
24 \pm 6 \\
\end{array}$ & $\begin{array}{l}90 \pm 11 \\
31 \pm 7 \\
20 \pm 5 \\
17 \pm 5\end{array}$ & $\begin{array}{c}351 \pm 18 \\
132 \pm 11 \\
79 \pm 8 \\
65 \pm 8\end{array}$ & $\begin{array}{c}189 \pm 13 \\
79 \pm 8 \\
51 \pm 7 \\
38 \pm 6 \\
\end{array}$ & $\begin{array}{c}129 \pm 11 \\
54 \pm 7 \\
32 \pm 5 \\
24 \pm 5 \\
\end{array}$ \\
\hline & \multicolumn{3}{|c|}{ Low $P$} & \multicolumn{3}{|c|}{ High lum } \\
\hline $\begin{array}{l}r_{1}=10 \mathrm{~mm} \\
r_{1}=15 \mathrm{~mm} \\
r_{1}=20 \mathrm{~mm} \\
r_{1}=25 \mathrm{~mm}\end{array}$ & $\begin{array}{c}\frac{1327}{145 \pm 7} \\
77 \pm 5 \\
59 \pm 5\end{array}$ & $\begin{array}{l}\frac{1731}{77 \pm 5} \\
46 \pm 4 \\
33 \pm 3\end{array}$ & $\begin{array}{c}410 \\
50 \pm 4 \\
29 \pm 3 \\
20 \pm 3\end{array}$ & $\begin{array}{l}\frac{4460}{490} \\
67 \pm 4 \\
48 \pm 4\end{array}$ & $\begin{array}{c}\frac{2807}{70 \pm 5} \\
35 \pm 3 \\
28 \pm 3\end{array}$ & $\begin{array}{l}\frac{1523}{40 \pm 3} \\
24 \pm 3 \\
18 \pm 2\end{array}$ \\
\hline
\end{tabular}

(iii) $\left(r_{1}=15 \mathrm{~mm}, B=3 \mathrm{~T}\right)$ and $\left(r_{1}=10 \mathrm{~mm}, B=\right.$ $5 \mathrm{~T})$ except for inflationary cases.

Figure 11 shows the $\left(P_{t}, \theta\right)$ plane for IPC electrons reaching the VD for $r_{1}=15 \mathrm{~mm}$ and $B=3,4,5 \mathrm{~T}$ and the evolution of the accumulation limit compared to the case with nominal parameters (illustrated by the thick line). One can see that the nominal and low $Q$ designs lead to the same results, both for the cross section and for the distance between the VD acceptance and the pair accumulation region. The large $Y$ design offers the safest distance to the accumulation region while for the low $P$ and high lum designs, the acceptance of the VD at 3 and $4 \mathrm{~T}$ is too close to the accumulation zone, not to say inside, as it is clearly shown in the last picture of Fig. 11. For this last design, the choice of $B=3 \mathrm{~T}$ is probably excluded if an innermost VD layer of $15 \mathrm{~mm}$ is desired. One notes that the low $P$ and high lum designs have their accumulation stripes moved significantly above the nominal case. This is due to a lower $\sigma_{z}$ and a lower $\sigma_{x}$ (see Table I), which result in larger deflection angles for the IPC electrons [1].

Finally, Table VII summarizes the main results concerning the IPC and the background in the VD. The integrated luminosities, $\mathcal{L}$, are extrapolated from the luminosities per bunch crossing, $\mathcal{L}_{b c}$, given by GUINEA-PIG. $N_{I P C e} / b c$ is the number of particles generated by the IPC processes and it depends both on the luminosity per bunch crossing, $\mathcal{L}_{b c}$, and the beamstrahlung radiation, quantified in VII by $N_{\gamma}$, the average number of emitted photons per beam particle. One sees that the virtual-virtual process is independent of the choice of beam parameters. The last part of Table VII gives an estimation of the number of IPC background particles reaching the VD for different inner layer radii and magnetic fields. Considering, for example, the TESLA
VD design, i.e. $r_{1}=15 \mathrm{~mm}$ and $B=4 \mathrm{~T}$, with the nominal, low $Q$ and large $Y$ beam parameter sets, $(\simeq$ $\left.1.2 \times 10^{6}\right) / \mathrm{s} \mathrm{IPC}$ particles are estimated to reach the VD, while for the low $P$ and high luminosity designs the rates increase to 1.5 and $\left(3.3 \times 10^{6}\right) / \mathrm{s}$, respectively.

\section{CONCLUSIONS}

The goals of this study were on the one hand to evaluate the uncertainty in the background from incoherent pair creation reaching the VD directly, predicted by the beambeam interaction simulation programs, GUINEA-PIG and CAIN, and on the other hand to evaluate the impact of the choice of beam parameters on this background.

The incoherent pair creation cross section predicted in CAIN is $12 \%$ less than the one in GUINEA-PIG. This comes from the processes involving virtual photons, BreitWheeler and Landau-Lifshitz processes, and is explained by the different choices in the two programs for the maximal value of the photon virtuality (set to the electron mass in CAIN and to half of the invariant mass of the produced final state in GUINEA-PIG). These differences are enhanced for the fraction of the events which produce particles reaching the VD directly, with CAIN predicting a $40 \%$ lower rate than GUINEA-PIG. A comparison with an $e^{+} e^{-} \rightarrow 4 f$ dedicated generator, $\mathrm{BDK}$, was made for the Landau-Lifshitz process and showed a very good agreement between its results and those of GUINEA-PIG. For the VD background arising from this process, CAIN predicts a 3 times lower rate then BDK. This difference comes indeed from the choice of virtuality limit since the three simulations leads to almost exactly the same results at low virtualities. 

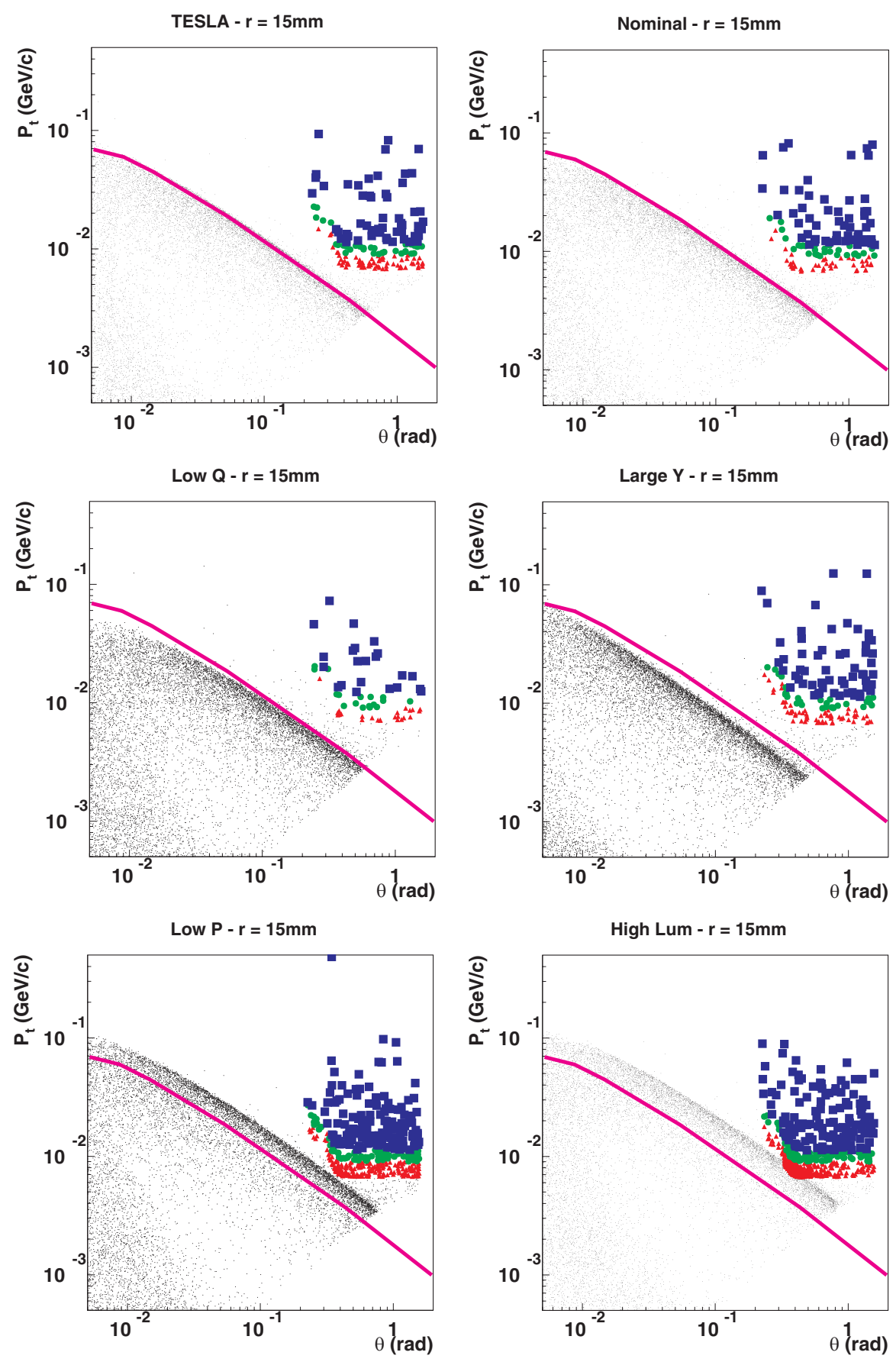

FIG. 11. (Color) $P_{t}$ versus $\theta$ plane for the background electrons reaching the VD for $r_{1}=15 \mathrm{~mm}$. The black points are all the IPC particles, generated including the beam-size effect (see Sec. II). Blue square-shaped points correspond to the background in the VD for a magnetic field of $5 \mathrm{~T}$. Adding the green circle-shaped (red triangle-shaped) ones, it corresponds to the background for $4 \mathrm{~T}$ ( $3 \mathrm{~T}$ ). The thick line indicates the edge of accumulation limit for the nominal design.

The similarity of the GUINEA-PIG and BDK virtuality spectra found, added to the almost equal cross-section predictions, seem to justify a choice of virtuality limit larger than the electron mass, as has been done in GUINEA-PIG. This gives some confidence in the results from that program, even if the particular value chosen does not have a first principle explanation and the BDK calculation does not include any radiative corrections.

Other potential uncertainties in the IPC rates predicted may arise from the two different sources of virtual-photon suppression, which are modeled with effective methods in GUINEA-PIG and CAIN, because neither corresponds to a 
TABLE VII. GUINEA-PIG simulation results of background production for different ILC design optimizations. The TESLA TDR case is shown for reference.

\begin{tabular}{|c|c|c|c|c|c|c|}
\hline & TESLA TDR & Nominal & Low $Q$ & Large $Y$ & Low $P$ & High lum \\
\hline \multicolumn{7}{|c|}{ Luminosities } \\
\hline $\mathcal{L}_{b c}\left[\mu \mathrm{b}^{-1}\right]$ & 1.92 & 1.46 & 0.71 & 1.14 & 2.84 & 3.44 \\
\hline $\mathcal{L}\left[n \mathrm{~b}^{-1} \mathrm{~s}^{-1}\right]$ & 27.0 & 20.6 & 20.0 & 16.1 & 18.9 & 48.5 \\
\hline \multicolumn{7}{|c|}{ IPC particles } \\
\hline$N_{\gamma}$ & 1.66 & 1.35 & 0.86 & 2.00 & 1.97 & 1.89 \\
\hline$N_{I P C e} / b c$ & 135200 & 96600 & 38100 & 96800 & 219000 & 258600 \\
\hline$\sigma_{B W} / b c[\mathrm{mb}]$ & 1.5 & 1.3 & 0.5 & 2.5 & 1.5 & 1.3 \\
\hline$\sigma_{B H} / b c[\mathrm{mb}]$ & 41 & 36 & 26 & 51 & 48 & 47 \\
\hline$\sigma_{L L} / b c[\mathrm{mb}]$ & 29 & 29 & 28 & 31 & 28 & 27 \\
\hline \multicolumn{7}{|c|}{ IPC particles reaching the VD } \\
\hline$N_{I P C e} / b c$ & $163 \pm 13$ & $128 \pm 11$ & $64 \pm 8$ & $150 \pm 12$ & $412 \pm 20$ & 1700 \\
\hline$N_{I P C e} /$ train $\left[10^{3}\right]$ & 460 & 362 & 360 & 424 & 548 & $\overline{4753}$ \\
\hline \multicolumn{7}{|c|}{$r_{1}=15 \mathrm{~mm} \quad B=4 T$} \\
\hline$N_{I P C e} / b c$ & $94 \pm 10$ & $86 \pm 9$ & $39 \pm 6$ & $90 \pm 9$ & $220 \pm 15$ & $240 \pm 15$ \\
\hline$N_{I P C e} /$ train $\left[10^{3}\right]$ & 265 & 243 & 220 & 254 & 291 & 679 \\
\hline \multicolumn{7}{|c|}{$r_{1}=15 \mathrm{~mm} \quad B=5 T$} \\
\hline$N_{I P C e} / b c$ & $58 \pm 8$ & $57 \pm 8$ & $22 \pm 5$ & $62 \pm 8$ & $142 \pm 12$ & $138 \pm 12$ \\
\hline$N_{I P C e} /$ train $\left[10^{3}\right]$ & 162 & 161 & 124 & 174 & 189 & 388 \\
\hline \multicolumn{7}{|c|}{$r_{1}=20 \mathrm{~mm} \quad B=3 T$} \\
\hline$N_{I P C e} / b c$ & $88 \pm 9$ & $85 \pm 9$ & $38 \pm 6$ & $90 \pm 9$ & $219 \pm 15$ & $230 \pm 15$ \\
\hline$N_{I P C e} /$ train $\left[10^{3}\right]$ & 249 & 239 & 212 & 254 & 291 & 650 \\
\hline \multicolumn{7}{|c|}{$r_{1}=20 \mathrm{~mm} \quad B=4 T$} \\
\hline$N_{I P C e} / b c$ & $52 \pm 7$ & $53 \pm 7$ & $22 \pm 5$ & $58 \pm 7$ & $131 \pm 11$ & $120 \pm 11$ \\
\hline$N_{I P C e} /$ train $\left[10^{3}\right]$ & 146 & 148 & 124 & 164 & 174 & 340 \\
\hline \multicolumn{7}{|c|}{$r_{1}=20 \mathrm{~mm} \quad B=5 T$} \\
\hline$N_{I P C e} / b c$ & $33 \pm 6$ & $31 \pm 6$ & $14 \pm 4$ & $36 \pm 6$ & $82 \pm 9$ & $83 \pm 9$ \\
\hline$N_{I P C e} /$ train $\left[10^{3}\right]$ & 92 & 86 & 80 & 103 & 110 & 233 \\
\hline
\end{tabular}

fully well-defined parameter region where standard methods can be applied. However, for what concerns the background in the VD, these two effects do not produce changes in rates larger than about $10 \%$, which is small in the present context.

The design of the accelerator can on the other hand significantly change the rates of direct pair backgrounds in the VD, for a given magnetic field and inner layer radius. Contrary to the nominal, low $Q$, and large $Y$ beam parameter sets, which leave the choices of magnetic field and VD innermost layer radius rather open, the low $P$ and high lum designs would be more constraining. For instance, for the case of the high lum design, to have similar background rates as with the other designs, a magnetic field of $5 \mathrm{~T}$ and a larger inner layer radius would have to be used.

\section{ACKNOWLEDGMENTS}

We would like to thank Toshiaki Tauchi and Kaoru Yokoya for the constructive discussions regarding the photon virtuality question. This work is supported by the
Commission of the European Communities under the 6th Framework Programme "Structuring the European Research Area," Contract No. RIDS-011899.

[1] D. Schulte, Ph.D. thesis, University of Hamburg, 1996 (Report No. TESLA-97-08).

[2] K. Yokoya, "User's Manual of CAIN-Version 2.3," 2001, http://www-acc-theory.kek.jp/members/cain/default.html

[3] F. A. Berends, P. H. Daverveldt, and R. Kleiss, Comput. Phys. Commun. 40, 285 (1986).

[4] Report No. ILC WG1, 2005, http://www-project.slac. stanford.edu/ilc/acceldev/beamparameters.html

[5] TESLA Technical Design Report No. DESY 2001-011, 2001.

[6] http://www-sid.slac.stanford.edu/

[7] http://www-jlc.kek.jp/

[8] K. Yokoya and P. Chen, KEK Report No. 91-2, 1991, pp. 1-38.

[9] G. L. Kotkin, V. G. Serbo, and A. Schiller, Int. J. Mod. Phys. A 7, 4707 (1992). 